\title{
A New Theory of Light
}

\author{
Nabeel Fattah \\ School of Electrical Electronic Engineering, Newcastle University, Newcastle, UK \\ Email: n.fattah@ncl.ac.uk, nabil.fatah@gmail.com
}

Received 26 February 2015; accepted 13 March 2015; published 17 March 2015

Copyright (C) 2015 by author and Scientific Research Publishing Inc.

This work is licensed under the Creative Commons Attribution International License (CC BY).

http://creativecommons.org/licenses/by/4.0/

(c) () Open Access

\begin{abstract}
Fundamentally, in the past, photons were thought to deliver light and other electromagnetic radiation, with dual physical properties, namely, waves and particles. However, in reality the theory of photons did not answer all the questions about electromagnetic waves. A new theory of light propagation, which in this study was named the seaton theory, might in the future be able to answer the questions about light propagation and electromagnetic waves. Seaton theory more accurately redefines electromagnetic radiation delivery by a new interpretation. Moreover, a further unique contribution of this study is that the electromagnetic field produced from the coil antenna can be seen if it is oscillated in the range of light frequency.
\end{abstract}

\section{Keywords}

\section{Photon, Electromagnetic Radiation, Light Propagation, Seaton}

\section{Introduction}

The form of electromagnetic radiation and the quantum of light is an elementary particle known as a photon. The photon can be defined as a major part of light delivery. Moreover, it has dual physical properties of waves and particles, and it is massless [1]. Certain discoveries have revealed a close relation among different types of light, such as X-rays, but this energy has higher electromagnetic radiation [2]. It was previously thought that the elementary particle did not require a medium to pass, and that photons did not need this medium to deliver light [3]. However, even though photons may be described as having duality, this idea does not fully explain the problem of where all the photons emerge from and does not answer all of the questions about light transition. For example, in an electromagnetic field produced by coil inductance, how is the electromagnetic field transmitted and where does it come from?

\section{Theory}

First of all, there is no free space in the universe. The femto particles in this study are coined seaton elementary 
particles, and these fill each point in the world [4]. These particles fundamentally help deliver light simply by sequentially passing the light in between them. Light is electromagnetic radiation and needs a medium through which to pass; in this study, this medium is a seaton, which is in contrast to the previous idea thatlightdoes not need a medium. Figure 1 shows the basic concept of seaton particles.

Seatons are everywhere, in air, water and materials. They basically support the propagation of light and all forms of electromagnetic radiation. There are spaces between each particle, and these particles start to vibrate when they receive electromagnetic power from a light source. The vibration is three dimensional oscillation, and causes the particles to collide with neighbouring particles, so that power then passes between them. This is described in Figure 2.

As shown in Figure 3, the magnetic field of the Earth [6] expands outside the atmosphere, and the seaton is responsible for delivering the field inside the Earth, both inside and outside the atmosphere. Previously, this was known as free space. In Figure 4, we can see that the seaton also carries and transmits the magnetic field of the connectors.

Overall, seaton particles cover the universe everywhere and there is no free space in between the Earth and

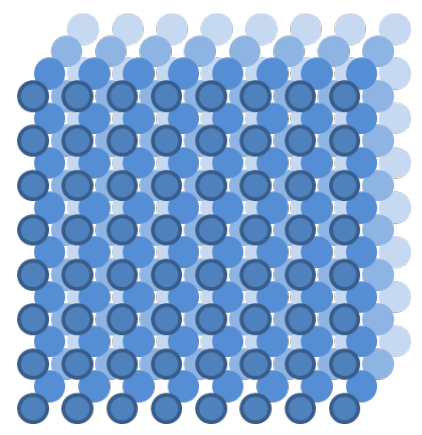

Figure 1. Seaton particles.

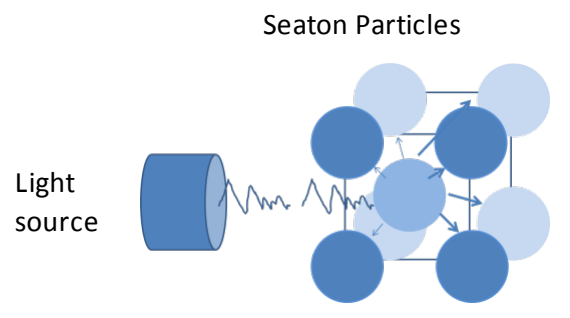

Figure 2. Particles oscillating strongly in light propagation directions.

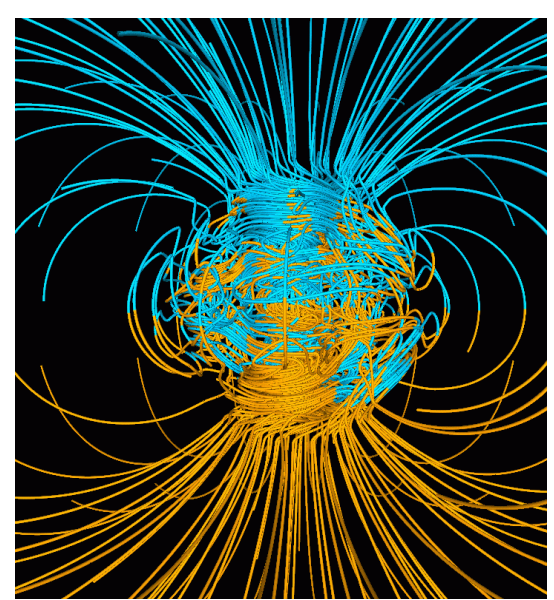

Figure 3. The Earth's magnetic field [5]. 


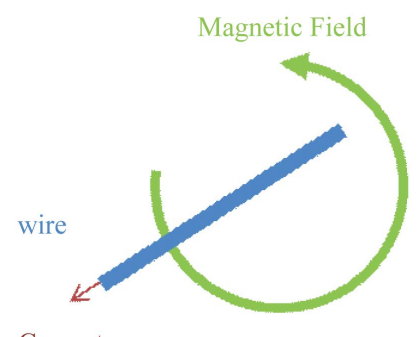

Current

Figure 4. Magnetic field of the single wire.

other planets, or inside a light bulb. That is, when the air is taken out of a light bulb, these particles remain. In the future, the existence of these particles may be demonstrated when the coil inductor is oscillated at $500 \mathrm{THz}$ frequency, so that the electromagnetic field is visible [7]. The seaton will deliver the field from one side of the coil to the other. In addition, any connectors (straight wires or any other coil shapes), if tuned at visible frequency ranges, will allow the field to become visible.

\section{Conclusion}

Light propagation and other electromagnetic waves generated from electronic devices are delivered by seaton particles. These particles are everywhere in the universe, including the air, sea and materials, but at different intensities. They carry and deliver light and other electromagnetic radiation. On this basis, seaton theory may resolve unanswered questions regarding electrometric propagation. If this happens, it will be seen that there is no free space in the world, and that photons do not move from the light source to the target. Instead, what may be seen is that seaton particles deliver the light.

\section{References}

[1] Moses, H.E. (1973) Physical Review A, 8, 1710-1721.

[2] Cowley, J.M. (1950) Journal of Applied Physics, 21, 24-30. http://dx.doi.org/10.1063/1.1699415

[3] Mikhail Vasilyev, K.S. (2000) Matter and Man. University Press of the Pacific, Honolulu.

[4] Vigier, J.P. (1990) IEEE Transactions on Plasma Science, 18, 64-72. http://dx.doi.org/10.1109/27.45506

[5] Wikipedia. (2015) Earth's Magnetic Field. http://en.wikipedia.org/wiki/Earth\%27s magnetic field

[6] Kern, J.W. and Vestine, E.H. (1963) Space Science Reviews, 2, 136-171.

[7] Shen, L., Tang, W., Xiang, H. and Zhuang, W. (2015) Microwave and Optical Technology Letters, 57, 424-427. http://dx.doi.org/10.1002/mop.28859 